\title{
Reliability Assessment Based on Multivariate Degradation Measures and Competing Failure Analysis
}

\author{
Zhu Liang, Yan Jun, Ling Haifeng \& Su Zhenglian \\ Engineering Institute of Engineering Corps, PLA University of Science and Technology \\ Nanjing 210007, China \\ E-mail: javis@qq.com
}

Received: October 11, 2011

Accepted: October 26, $2011 \quad$ Published: December 1, 2011

doi:10.5539/mas.v5n6p232

URL: http://dx.doi.org/10.5539/mas.v5n6p232

\begin{abstract}
An reliability assessment model based on multivariate degradation measures and competing failure analysis is presented. The multi-dimensionality reduction processing has applied to multivariate degradation measures to extract the key features for ease of calculation. The cumulative distribution function of sudden failure is calculated in terms of degradation data rather than time domains, and the reliability assessment model based on competing failure analysis is established. An example of an equipment component with competing failure data is applied to verify the validate of the model.
\end{abstract}

Keywords: Multivariate degradation measures, Sudden failure, Competing failure, Reliability

\section{Introduction}

Equipment component failure is usually caused by the competition between sudden failure and degradation failure. There have been many literatures (Nelson W B., 1975; Bai D S, Chun Y R., 1991; Zhang Z H, Mao S S., 1995; Wang B X., 2002) for the study of reliability assessment on competing failure mechanism, used the failure rate to build the completion failure assessment model. Couallier (Nikulin M. Probability, 2006) described the relationship between the rate of sudden failure and degradation using exponential function, and Zhao Jianyin(Zhao J Y, Liu F, Sun Q, et al., 2006) assumed linear function to describe the relationship. Yang and Xue(Yang K, Xue J., 1996) make a simple analysis for the concomitant circumstance which have degradation failure and sudden failure. Zuo(Zuo M J , Jiang R, Yan R CM., 1999) has made improvement to their analysis method on the basis of summing up the work of Yang and Xue, but the model is too simplified to have strong applicability. Huang(Huang W, Askin R G., 2003) assume that sudden failure and degradation failure independent of each other, then established a competing failure model using series model and two failure rate functions.

The research in those literatures has some limitations. On one hand, the current research on reliability assessment method for degradation failure are mostly based on single degradation measure, but these studies are difficult to apply to reality because many components has multivariate degradation measure. On the other hand, you cannot simply assume that sudden failure and degradation failure independent of each other, because the more serious performance degradation, the more likely to occur sudden failure.In addition, it is difficult to record the exact sudden failure time in a scheduled observation experiment, so obtain accurate failure rate model is impossible, but the degradation are readily available while occur sudden failure.

Based on the above analysis and the new assumptions, the paper presents a reliability analysis model based on multivariate degradation measures and competing failure, process multivariate data using multi-dimensionality reduction method, describe the cumulative distribution function of sudden failure in terms of degradation data, and the reliability assessment model based on competing failure analysis is established, finally some examples are given.

\section{Assumptions}

This study builds on the following assumptions:

(1) Component failure modes with $p$ kinds of degradation and a sudden failure mode, the two çompete with each other leading to product failure. Degraded feature vector $Y(t)=\left[Y_{1}(t), Y_{2}(t), \cdots, Y_{p}(t)\right]^{\prime}$ is made up of $p$ degradation characteristics. 
(2) Components performance with running time is reduced gradually, the degradation process is irreversible. Degradation characteristics of $Y_{k}(t)$ is an increasing function or decreasing function of time $t$, this paper assumes that $Y_{k}(t)$ is an increasing function of $t$ without losing generality of cases.

(3)In different times $t$, the multiple performance degradation of components are subject to the same family of distributions $F_{Y}(y \mid t)$, this paper assumes that obey the multivariate normal distribution.

(4)Initial state of degradation are both zero, the degradation characteristics all have degradation failure threshold $y_{k s}$, the component judge failure when a degradation of $y_{k}(t)$ is higher than $y_{k s}$.

(5)Assumed sudden failure is relevant with only one performance degradation characteristics.

\section{Reduction analysis of multivariate degradation measures}

Component has $p$ types of degradation failure model, its general expression for reliability is

$$
R_{s}(t)=\int_{0}^{y_{p s}} \cdots \int_{0}^{y_{2 s}} \int_{0}^{y_{1 s}} f\left(y_{1}, \cdots, y_{p}\right) d y_{1} d y_{2} \cdots d y_{p}
$$

Where $f\left(y_{1}, \cdots, y_{p}\right)$ is the joint probability density function of $p$ performance degradation measures in time $t$. The problem of solving $f\left(y_{1}, \cdots, y_{p}\right)$ is the core problem. If these characteristics are independent of each other, the calculations of equation (1) can be simplified, then equation (1) can be transformed into

$$
R_{s}(t)=\int_{0}^{y_{1 s}} f\left(y_{1}\right) d y_{1} \cdots \int_{0}^{y_{p s}} f\left(y_{p}\right) d y_{p}=\prod_{k=1}^{p} R\left(y_{k}(t)\right)
$$

The degradation characteristics tend to restrict each other in the process of components degradation. Some degradation characteristics have a strong correlation between, and some have a weak correlation. To simplify the calculation, the performance degradation can be multi-dimensional data reduction process, the aim is transform the related multiple performance degradation data into unrelated data. The steps are classification and filtering.

(1) Classification of multivariate degradation measures. Principal component analysis can be used based on related degree of degradation characteristics classification.

(2) Filtering. The time function of degradation $\tilde{g}_{k}(t, \theta)$ can be obtained using statistical fitting method, then pseudo-failure time can be calculated based on $\tilde{T}_{k s}=\tilde{g}_{k}^{-1}\left(y_{k s}, \theta\right)$. The higher of $\tilde{T}_{k s}$, the more slowly degraded of the $\mathrm{k}$-th degradation, otherwise degraded faster. If degradation of certain degradation characteristics significantly slower, these characteristics have little impact on reliability and can be removed. For the remaining degradation characteristics can be filtered in characteristics with strong related: Select a key degradation characteristics which had the fastest degraded to represent these strong related characteristics according to the comparison of these pseudo-failure time.

$b$ dimension degradation data can be considered relatively independent after classification and filtering of multivariate degradation measures, then the reliability expression of component is

$$
R_{s}(t)=\prod_{k=1}^{b} R\left(y_{k}(t)\right)
$$

\section{The reliability assessment model based on competing failure}

For the sudden failure, set the failure rate of $\lambda_{h}(t \mid x)$ when the amount of degradation is $x$, the component performance degradation is $x(t)$ when time $t, T_{h}$ for the time of sudden failure, then the reliability function of sudden failure is

$$
R_{h}(t \mid x)=\exp \left[-\int_{0}^{t} \lambda_{h}(\tau \mid x) d \tau\right]
$$

Where $\lambda_{h}(\tau \mid x)$ is the failure rate function of sudden failure when the amount of degradation is $x, \tau$ is the integral variable of $t$.

If sudden failure and all degradation are independent, the reliability function competing failure is 


$$
\begin{aligned}
& R_{c}(t)=P\left(\min \left[T_{1 s}, \cdots, T_{b s}, T_{h}\right]>t\right)=P\left(\min \left[T_{1 s}, \cdots, T_{b s}\right]>t, T_{h}>t\right) \\
& =R_{h}(t) R_{s}(t)=\exp \left[-\int_{0}^{t} \lambda_{h}(\tau) d \tau\right] \prod_{k=1}^{b} R\left(y_{k}(t)\right)
\end{aligned}
$$

In General, the more serious performance degradation, the more likely to occur sudden failure, so it cannot simply assume that sudden failure and degradation failure independent of each other. According to the chart analysis of the test data, sudden failure can be considered relevant with degradation if more and more sudden failure in the test during the process of degradation.

Assumed sudden failure is relevant with only one performance degradation characteristics in assumption (5). Due to the degradation of weight distribution is a function of time, thus can avoid the direct relationship of failure rate and time, and using the degradation to characterize the regularity of sudden failure.

When the amount of the k-th component degradation is $y_{k}$, note sudden failure on degradation condition probability for the $R_{h}(t \mid x)=R_{h}\left(y_{k}\right)$, so

$$
\begin{aligned}
& R_{c}(t)=P\left(\min \left[T_{1 s}, \cdots, T_{b s}, T_{h}\right]>t\right)=P\left(\min \left[T_{1 s}, \cdots, T_{b s}\right]>t, T_{h}>t\right) \\
& =\int_{0}^{y_{1 s}} f\left(y_{1}\right) d y_{1} \cdots \int_{0}^{y_{k s}} R_{h}\left(y_{k}\right) f\left(y_{k}\right) d y_{k} \cdots \int_{0}^{y_{b s}} f\left(y_{b}\right) d y_{b}
\end{aligned}
$$

\section{Example analysis}

Suppose a total 22 samples for key components of equipment testing, there are 4 degradation characteristics $Y_{1}(t), Y_{2}(t), Y_{3}(t), Y_{4}(t)$, the fail threshold $y_{s}=[8,10,14,12]^{\prime}$. Test data once every 250 hours to 4000 hours so far.

(1) Reduction analysis of multivariate degradation measures

According to multiple performance degradation data failure threshold and correlation matrix, can determine the key degradation characteristics independent of each other are $Y_{1}(t)$ and $Y_{2}(t)$ by the application of principal component test methods and similarity analysis. The specific methods and steps can be found in literature (Zhong Q H, Zhang Z H , Liang S J., 2011), this does not repeat.

(2)Estimates the parameters of the Distribution function of performance degradation

Table 1 shows the data of $Y_{1}(t)$ in each $500 \mathrm{~h}$ intervals, samples of which 15 performance degradation occurs, the other 7 samples of unexpected failure.

The degradation expectations in $t_{k}$ is $\mu\left(t_{k}, \alpha\right)=\bar{x}_{k}=\left(\sum_{n=1}^{N} x_{n k}\right) / N$, the standard deviation of

$$
\sigma\left(t_{k}, \beta\right)=\sqrt{\frac{1}{N-1} \sum_{n=1}^{N}\left(x_{n k}-\bar{x}_{k}\right)^{2}}
$$

Where $\alpha$ and $\beta$ are parameter vectors, $\mathrm{N}$ is the number of samples, $x_{n k}$ is the amount of the $\mathrm{n}$-th degradation in time $t_{k}$. Computed the mean and standard deviation of the sample degradation, analyze the sample mean and standard deviation showed a linear trend, can be considered the degradation of the mean and standard deviation is the linear function of time. Set $\mu(t)=a t+b, \sigma(t)=c t+d$, using regression analysis and least-squares method based on sample data, get the estimate parameters of degenerate distribution function of $Y_{1}(t)$ as $\hat{a}_{1}=0.002, \hat{b}_{1}=0.0098, \hat{c}_{1}=0.0005, \hat{d}_{1}=0.0261$. Similarly, can get the estimate parameters of degenerate distribution function of $Y_{2}(t)$ as $\hat{a}_{2}=0.005, \hat{b}_{2}=0.0043, \hat{c}_{2}=0.0011, \hat{d}_{2}=0.0112$.

(3) Analysis on sudden failure

In experiment, the sudden failure respectively when the $1,21,17,3,11,7,14$, sample degradation respective 
reached $1.81,3.97,6.33,7.05,8.02,8.35,9.21$, its corresponding sudden failure reliability $R_{h}(x)$ were $21 /$ 22, $20 / 22,19 / 22,18 / 22,17 / 22,16 / 22,15 / 22$. Set the sudden failure reliability in this test obeys two-parameter Weibull distribution, that is $R_{h}(x)=\exp \left(-(x / \eta)^{\lambda}\right)$, where $\lambda$ and $\eta$ are shape parameter and scale parameter. Using the method of least squares estimate, can get $\hat{\lambda}=1.2373, \hat{\eta}=24.0601$.

(4) Solving the reliability of competing failure models

According to the proposed modeling method, get the reliability expression of key equipment components in the competing failure mode for

$$
\begin{aligned}
R_{c}(t)= & \int_{0}^{y_{1 s}} \exp \left[-\left(\frac{y_{1}}{\hat{\eta}}\right)^{\hat{\lambda}}\right] \frac{1}{\sqrt{2 \pi\left(\hat{c}_{1} t+\hat{d}_{1}\right)}} \exp \left\{-\frac{\left[y_{1}-\left(\hat{a}_{1} t+\hat{b}_{1}\right)\right]^{2}}{2\left(\hat{c}_{1} t+\hat{d}_{1}\right)^{2}}\right\} d y_{1} \\
& \times \int_{0}^{y_{2 s}} \frac{1}{\sqrt{2 \pi\left(\hat{c}_{2} t+\hat{d}_{2}\right)}} \exp \left\{-\frac{\left[y_{2}-\left(\hat{a}_{2} t+\hat{b}_{2}\right)\right]^{2}}{2\left(\hat{c}_{2} t+\hat{d}_{2}\right)^{2}}\right\} d y_{2}
\end{aligned}
$$

Separate each parameter calculated in the first two steps into the above equation, obtained the reliability at all times under competing failure mode, its reliability curves as shown in Figure 1. The following curve is considering competing failure reliability, curve above is only considering degradation of reliability of failure.

\section{Conclusion}

This paper studied the competition issues contains multiple performance degradation failure and sudden failure, simplified the calculations through the multivariate degradation data dimension reduction, the cumulative distribution function of sudden failure is calculated in terms of degradation data rather than time domains, finally get a concise and rational competition failure model. Studies have shown that only degradation failure model and ignore the sudden failure will overestimate the component reliability.

\section{References}

Bai, D. S., \& Chun, Y. R. (1991). Optimum Simple Step Stress Accelerated Life Tests with Competing Causes of Failure. IEEE: Trans Reliability(S0018-9529), 40(5), 622 627.

Huang, W., \& Askin, R. G.. (2003). Reliability analysis of electronic devices with multiple competing failure modes involving performance aging degradation. Quality and Reliability Engineering International, 19 (3), 241 254. http://dx.doi.org/10.1002/qre.524

Nelson, W. B. (1975). Graphical Analysis of Accelerated Life Test Data with a Mix of Failure Models. IEEE: Trans Reliability (S0018-9529). 24 (10), 230-237.

Nikulin, M. P. (2006). statistics and modeling in public health (73 89) [M] .New York: Springer-Verlag.

Wang, B. X. (2002). Statistical Analysis for Accelerated Life Tests with Competing Cuases of Failure . ACTA MATHEMATICAE APPLICATAE SINICA, 25(2), 254 262.

Yang, K., \& Xue, J. (1996). Continuous state reliability analysis[C ] // [ s. 1. ]: Proc. Annual Reliability and Maintainability Symposium, 251 257.

Zhang, Z. H., \& Mao, S. S. (1995). Constant Stress Accelerated Life Testing Statistical Analysis of Competing Failure under Exponential Distribution . Chinese Journal of Applied Probability and Statisties. 11(3), 289 296.

Zhao, J. Y., Liu, F., Sun, Q., et al. (2006). Reliability analysis of metallizedfilm pulse capacitor under competing failure modes. Systems Engineering-Theory \& Practice, 26 (1), 60 64.

Zhong, Q. H., Zhang, Z. H., \& Liang, S. J. (2011).Reliability analysis approach based on multivariate degradation data. Systems Engineering-Theory \&Practice, 31 (3), 544 551 ( In Chinese).

Zuo, M. J., Jiang, R., \& Yan, R. C. M. (1999). Approaches for reliability modeling of continuous-state device. IEEE Trans On Rel, 48 (1), 9 18. http://dx.doi.org/10.1109/24.765922 
Table 1. Degradation of the sample components with the record of sudden failure

\begin{tabular}{|c|c|c|c|c|c|c|c|c|c|c|c|c|c|c|c|c|c|}
\hline \multirow{2}{*}{ No } & \multicolumn{8}{|c|}{ Time (hour) } & \multirow{2}{*}{ No } & \multicolumn{8}{|c|}{ Time (hour) } \\
\hline & 500 & 1000 & 1500 & 2000 & 2500 & 3000 & 3500 & 4000 & & 500 & 1000 & 1500 & 2000 & 2500 & 3000 & 3500 & 4000 \\
\hline 1 & 0.88 & 1.81 & & & & & & & 12 & 1.49 & 3.00 & 4.50 & 6.26 & 7.80 & 8.93 & 10.45 & 12.21 \\
\hline 2 & 1.17 & 1.99 & 2.97 & 3.94 & 4.45 & 5.27 & 6.02 & 6.88 & 13 & 0.93 & 1.96 & 3.29 & 4.11 & 4.91 & 5.84 & 6.84 & 7.88 \\
\hline 3 & 0.96 & 2.39 & 3.52 & 4.77 & 6.15 & 7.05 & & & 14 & 0.99 & 2.37 & 3.35 & 4.61 & 6.14 & 7.51 & 9.21 & \\
\hline 4 & 1.22 & 2.30 & 3.75 & 4.99 & 6.07 & 7.16 & 8.42 & 9.28 & 15 & 1.07 & 1.77 & 2.40 & 3.02 & 3.75 & 4.76 & 5.46 & 6.24 \\
\hline 5 & 0.93 & 2.72 & 4.34 & 5.48 & 6.72 & 8.00 & 9.49 & 10.94 & 16 & 0.83 & 1.52 & 2.27 & 3.42 & 4.11 & 4.63 & 5.84 & 6.62 \\
\hline 6 & 0.92 & 1.46 & 2.39 & 2.94 & 4.09 & 4.84 & 5.57 & 7.17 & 17 & 1.02 & 2.50 & 3.87 & 4.92 & 6.33 & & & \\
\hline 7 & 0.80 & 1.95 & 3.17 & 4.58 & 6.09 & 7.39 & 8.35 & & 18 & 0.70 & 1.35 & 2.55 & 3.39 & 4.09 & 5.41 & 6.14 & 6.88 \\
\hline 8 & 1.39 & 2.86 & 3.81 & 5.35 & 6.71 & 8.61 & 9.95 & 11.01 & 19 & 0.74 & 1.85 & 2.95 & 3.92 & 5.47 & 6.50 & 7.39 & 8.09 \\
\hline 9 & 0.61 & 1.77 & 2.58 & 3.38 & 4.63 & 5.62 & 6.32 & 7.59 & 20 & 0.80 & 1.74 & 3.59 & 4.44 & 5.22 & 5.96 & 6.99 & 7.88 \\
\hline 10 & 0.62 & 1.95 & 2.95 & 3.79 & 4.50 & 4.98 & 5.61 & 6.14 & 21 & 0.87 & 2.41 & 3.66 & 3.97 & & & & \\
\hline 11 & 0.89 & 2.21 & 3.49 & 4.76 & 6.13 & 7.43 & 8.02 & & 22 & 1.00 & 1.96 & 2.84 & $4 . .01$ & 4.80 & 5.66 & 6.54 & 7.42 \\
\hline
\end{tabular}

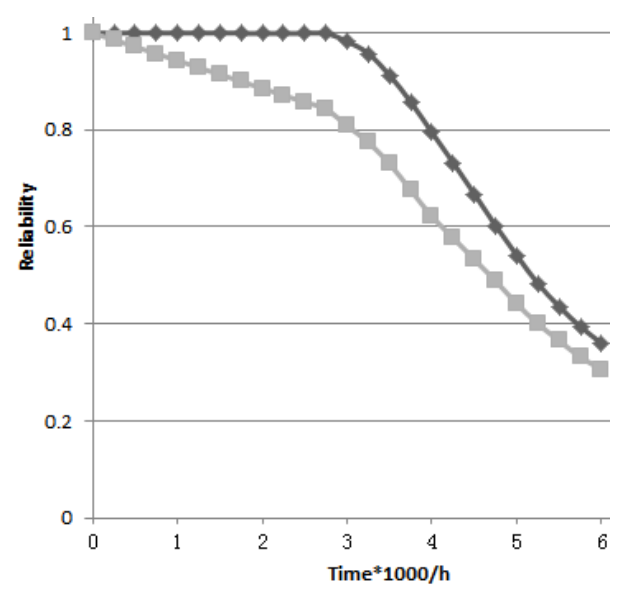

Figure 1. Reliability curves 